\title{
Synthesis and styrene copolymerization of novel fluoro, methyl, and phenoxy ring-disubstituted isobutyl phenylcyanoacrylates
}

Kaartikeya Gupta, Amna Farshori, Maya R. Fitzgerald, Rachel I. Lewandowski, Michael J. Mojarro, Alexis Petrauskas, Shailar K.Webber, Lucienna M. Wolf, Sara M. Rocus, William S. Schjerven and Gregory B. Kharas

DePaul University, Chemistry and Biochemistry Department, 1110 West Belden Avenue, Chicago, IL 60614-3214

\footnotetext{
ABSTRACT

Novel ring-disubstituted isobutyl phenylcyanoacrylates, $\mathrm{RPhCH}=\mathrm{C}(\mathrm{CN}) \mathrm{CO}_{2} \mathrm{CH}_{2} \mathrm{CH}\left(\mathrm{CH}_{3}\right)_{2}$ (where R is 2-fluoro-5-methyl, 2-fluoro-6-methyl, 3-fluoro-2-methyl, 3-fluoro-4-methyl, 4fluoro-2-methyl, 4-fluoro-3-methyl, 5-fluoro-2-methyl, 4-fluoro-3-phenoxy) were synthesized by the piperidine catalyzed Knoevenagel condensation of ring-disubstituted benzaldehydes and isobutyl cyanoacetate and characterized by $\mathrm{CHN}$ analysis, $\mathrm{IR},{ }^{1} \mathrm{H}$ and ${ }^{13} \mathrm{C}$ NMR. The acrylates were copolymerized with styrene in solution with radical initiation $(\mathrm{ABCN})$ at $70^{\circ} \mathrm{C}$. The compositions of the copolymers were calculated from nitrogen analysis.
} 
*Address correspondence to: Gregory B. Kharas, Chemistry and Biochemistry Department, DePaul University, Chicago, IL 60614-3214. Fax: 773-325-7421; E-mail:

gkharas@depaul.edu

\section{Introduction}

Cyanoacrylates is family of vinyl monomers renowned for their high reactivity, instant adhesive properties, and wide-ranging applications [1-3]. Trisubstituted ethylenes (TSE), ring-functionalized $\left(\mathrm{R}^{1}\right)$ alkyl $\left(\mathrm{R}^{2}\right)$ phenylcyanoacrylates, $\mathrm{R}^{1} \mathrm{PhCH}=\mathrm{C}(\mathrm{CN}) \mathrm{CO}_{2} \mathrm{R}^{2}(\mathrm{PCA})$ continue to attract attention as compounds with variety of applications [4-11]. Thus, methoxy ring-substituted methyl phenylcyanoacrylate, MPCA was used in synthesis of pyridotriazines and triazolopyridines [4]. Dimethylamino ring-substituted MPCA was examined among other cyanovinylheteroaromatics in relation to organic nonlinear optics [5]. There are a number of applications of ethyl phenylcyanoacrylate, EPCA and its ringsubstituted derivatives which include studies of catalysis [6] and potential antimicrobial and antioxidant agents [7]. 2,4-Dimethoxyphenyl EPCA was used in design, synthesis and study of anticancer activity of novel benzothiazole analogues [8], in synthesis of thiazacridine derivatives as anticancer agents against breast and hematopoietic neoplastic cells [9] and in DABCO-catalyzed Knoevenagel condensation using hydroxy ionic liquid as a promoter [10]. This EPCA was involved in catalysis study of N,N'dialkylimidazolium dimethyl phosphates [11], in synthesis and study of antimicrobial activity of some cyanoacrylates [12], as well as in synthesis of antiproliferative active 2aminobenzimidazole derivatives [13]. 
In regards to polymerization reactivity, previous studies showed that PCAs as all TSE monomers containing substituents larger than fluorine have very low reactivity in radical homopolymerization due to polar and steric reasons [14]. Although steric difficulties preclude homopolymerization of such monomers, their copolymerization with a monosubstituted alkenes makes it possible to overcome these steric problems. Thus, copolymerization of electrophylic TSE monomers having double bonds substituted with halo, cyano, and carbonyl groups and electron-rich monosubstituted ethylenes such as styrene, $N$-vinylcarbazole, and vinyl acetate [15-17] show a tendency toward the formation of alternating copolymers - thus suggesting a way of functionalization of commercial polymers via introduction of isolated monomer units in copolymers. Earlier we have reported synthesis and styrene copolymerization a number of methyl and oxy ringsubstituted PCAs, such esters as methyl [18,19], ethyl [20-22], propyl [23, 24], isopropyl [25-27], and butyl [28, 29].

Our objectives in exploration of novel isobutyl phenylcyanoacrylates (IPCA) were twofold: (1) to utilize Knoevenagel condensation for synthesis of IPCA compounds with a variety of potentially reactive functional groups; (2) to explore feasibility of radical copolymerization with a commercial monomer styrene.

Thus, in continuation of our investigation of novel PCA compounds we have prepared oxy ring-disubstituted isobutyl PCA, $\mathrm{RPhCH}=\mathrm{C}(\mathrm{CN}) \mathrm{CO}_{2} \mathrm{CH}_{2} \mathrm{CH}\left(\mathrm{CH}_{3}\right)_{2}$, where $\mathrm{R}$ is 2-fluoro-5methyl, 2-fluoro-6-methyl, 3-fluoro-2-methyl, 3-fluoro-4-methyl, 4-fluoro-2-methyl, 4fluoro-3-methyl, 5-fluoro-2-methyl, 4-fluoro-3-phenoxy, and explored the feasibility of their 
copolymerization with styrene. To the best of our knowledge, there have been no reports on either synthesis of these compounds, nor their copolymerization with styrene.

\section{Experimental}

\subsection{Materials}

2-Fluoro-5-methyl, 2-fluoro-6-methyl, 3-fluoro-2-methyl, 3-fluoro-4-methyl, 4-fluoro-2methyl, 4-fluoro-3-methyl, 5-fluoro-2-methyl, 4-fluoro-3-phenoxy benzaldehydes, isobutyl cyanoacetate, piperidine, styrene, 1,1'-azobis(cyclohexanecarbonitrile) (ABCN), and toluene supplied from Sigma-Aldrich Co., were used as received.

\subsection{Instrumentation}

Infrared spectra of the IPCA compounds and polymers ( $\mathrm{NaCl}$ plates) were determined with an ABB FTLA 2000 FT-IR spectrometer. The melting points of the IPCA compounds and the glass transition temperatures $\left(T_{\mathrm{g}}\right)$, of the copolymers were measured with TA (Thermal Analysis, Inc.) Model Q10 differential scanning calorimeter (DSC). The thermal scans were performed in a 25 to $150^{\circ} \mathrm{C}$ range on second heat at heating rate of $10^{\circ} \mathrm{C} / \mathrm{min}$. $T_{\mathrm{g}}$ was taken as a midpoint of a straight line between the inflection of the peak's onset and endpoint. The thermal stability of the copolymers was measured by thermogravimetric analyzer (TGA) TA Model Q50 from ambient temperature to $800^{\circ} \mathrm{C}$ at $20^{\circ} \mathrm{C} / \mathrm{min}$ in the flow of nitrogen $(20$ $\mathrm{mL} / \mathrm{min}$ ). The molecular weights of the polymers was determined relative to polystyrene standards in THF solutions with sample concentrations $0.8 \%(\mathrm{w} / \mathrm{v})$ by gel permeation chromatography (GPC) using a Altech 426 HPLC pump at an elution rate of $1.0 \mathrm{~mL} / \mathrm{min}$; Phenogel $5 \mu$ Linear column at $25^{\circ} \mathrm{C}$ and Viscotek $302 \mathrm{RI}$ detector. ${ }^{1} \mathrm{H}$ and ${ }^{13} \mathrm{C}$ NMR spectra 
were obtained on 10-25\% (w/v) IPCA or polymer solutions in $\mathrm{CDCl}_{3}$ at ambient temperature using Avance $300 \mathrm{MHz}$ spectrometer. CHN-elemental analyses of IPCA compounds and nitrogen analysis of the copolymers were performed by Midwest Microlab, LLC (IN).

\section{Results and discussion}

\subsection{Synthesis and characterization of isobutyl phenylcyanoacrylates}

All isobutyl phenylcyanoacrylates (IPCA) compounds were synthesized by Knoevenagel condensation [31] of appropriate benzaldehydes with isobutyl cyanoacetate, catalyzed by base, piperidine (Scheme 1).

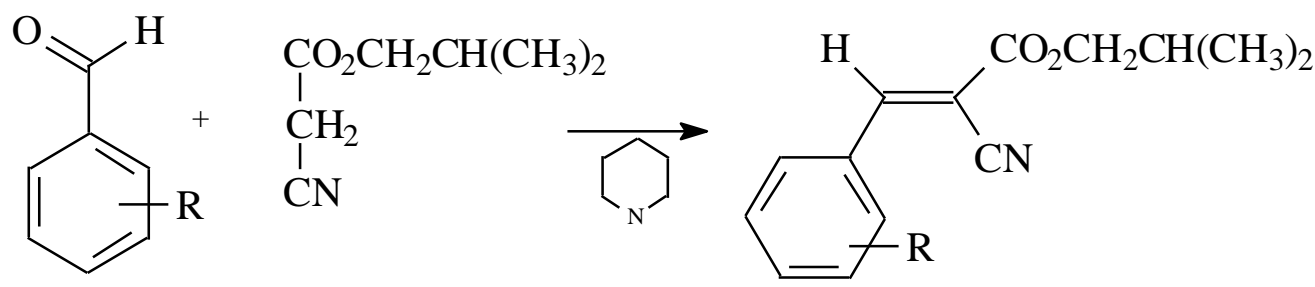

Scheme 1. Synthesis of isobutyl R-phenylcyanoacrylates, where R is 2-fluoro-5-methyl, 2fluoro-6-methyl, 3-fluoro-2-methyl, 3-fluoro-4-methyl, 4-fluoro-2-methyl, 4-fluoro-3methyl, 5-fluoro-2-methyl, 4-fluoro-3-phenoxy.

The preparation procedure was essentially the same for all the monomers. In a typical synthesis, equimolar amounts of isobutyl cyanoacetate and an appropriate benzaldehyde were mixed in equimolar ratio in a $20 \mathrm{~mL}$ vial. A few drops of piperidine were added with stirring. The reactions was allowed to proceed $48 \mathrm{hrs}$ at r.t. The product of the reaction was 
isolated by filtration and purified by crystallization from 2-propanol. The condensation reaction proceeded smoothly, yielding products, which were purified by conventional techniques. Melting points of the compounds in crystalline state were measured by DSC.

The compounds were characterized by IR, ${ }^{1} \mathrm{H}$ and ${ }^{13} \mathrm{C}$ NMR spectroscopies. No stereochemical analysis of the novel ring-substituted IPCA was performed since no stereoisomers ( $E$ or/and $Z$ ) of known configuration were available.

\subsubsection{Isobutyl 2-fluoro-5-methylphenylcyanoacrylate}

Yield: $85.2 \%$; ${ }^{1} \mathrm{H}$ NMR: $\delta 8.5(\mathrm{~s}, 1 \mathrm{H}, \mathrm{CH}=), 8.2-6.9(\mathrm{~m}, 3 \mathrm{H}, \mathrm{Ph}), 4.1\left(\mathrm{~d}, 2 \mathrm{H}, \mathrm{CH}_{2}\right), 2.4(\mathrm{~s}$, $\left.3 \mathrm{H}, \mathrm{CH}_{3}\right), 2.1(\mathrm{~m}, 1 \mathrm{H}, \mathrm{CH}), 1.0\left(\mathrm{~d}, 6 \mathrm{H}, \mathrm{CH}_{3}\right) ;{ }^{13} \mathrm{C} \mathrm{NMR}: \delta 162(\mathrm{C}=\mathrm{O}), 158(\mathrm{HC}=), 146$ 136, 135, 129, $120(\mathrm{Ph}), 116(\mathrm{CN}), 104(\mathrm{C}=), 73\left(\mathrm{CH}_{2}\right), 27(\mathrm{CH}), 21\left(\mathrm{PhCH}_{3}\right), 19\left(\mathrm{CH}_{3}\right)$; IR: (cm-1) 3129-2760 (m, C-H), 2226 (m, CN), 1732 (s, C=O), 1610 (s, C=C), 1227 (s, CO-CH 3 ), 827, 754 (s, C-H out of plane). Anal. calcd. for $\mathrm{C}_{15} \mathrm{H}_{16} \mathrm{FNO}_{2}$ : C, 68.95; H, 6.17; N, 5.36; Found: C, 65.32; H, 6.26; N, 5.66.

\subsubsection{Isobutyl 2-fluoro-6-methylphenylcyanoacrylate}

Yield 91.4\%; ${ }^{1} \mathrm{H}$ NMR: $\delta 8.3(\mathrm{~s}, 1 \mathrm{H}, \mathrm{CH}=), 7.4-6.9(\mathrm{~m}, 3 \mathrm{H}, \mathrm{Ph}), 4.1\left(\mathrm{~d}, 2 \mathrm{H}, \mathrm{CH}_{2}\right), 2.4(\mathrm{~s}$, $\left.3 \mathrm{H}, \mathrm{CH}_{3}\right), 2.1(\mathrm{~m}, 1 \mathrm{H}, \mathrm{CH}), 1.0\left(\mathrm{~d}, 6 \mathrm{H}, \mathrm{CH}_{3}\right) ;{ }^{13} \mathrm{C} \mathrm{NMR}: \delta 162(\mathrm{C}=\mathrm{O}), 156(\mathrm{HC}=), 149$, 133, 112, $111(\mathrm{Ph}), 116(\mathrm{CN}), 110(\mathrm{C}=), 72\left(\mathrm{CH}_{2}\right), 28(\mathrm{CH}), 23\left(\mathrm{CH}_{3}\right), 19\left(\mathrm{CH}_{3}\right)_{2}$; IR: $\left(\mathrm{cm}^{-1}\right)$ 3097-2876 (m, C-H), 2231 (m, CN), 1730 (s, C=O), 1617 (s, C=C), 1267 (s, C-O$\mathrm{CH}_{3}$ ), 783 (s, C-H out of plane). Anal. calcd. for $\mathrm{C}_{15} \mathrm{H}_{16} \mathrm{FNO}_{2}$ : C, 68.95; H, 6.17; N, 5.36; Found: C, 64.5; H, 6.26; N, 5.71.

\subsubsection{Isobutyl 3-fluoro-2-methylphenylcyanoacrylate}


Yield 82.8\%; ${ }^{1} \mathrm{H}$ NMR: $\delta 8.5(\mathrm{~s}, 1 \mathrm{H}, \mathrm{CH}=), 7.9-7.0(\mathrm{~m}, 3 \mathrm{H}, \mathrm{Ph}), 4.2\left(\mathrm{~d}, 2 \mathrm{H}, \mathrm{CH}_{2}\right), 2.4(\mathrm{~s}$,

$\left.3 \mathrm{H}, \mathrm{PhCH}_{3}\right), 2.1(\mathrm{~m}, 1 \mathrm{H}, \mathrm{CH}), 1.0\left(\mathrm{~d}, 6 \mathrm{H}, \mathrm{CH}_{3}\right) ;{ }^{13} \mathrm{C} \mathrm{NMR}: \delta 166(\mathrm{C}=\mathrm{O}), 152(\mathrm{HC}=)$, 128, 127, 125, $124(\mathrm{Ph}), 116(\mathrm{CN}), 106(\mathrm{C}=), 73\left(\mathrm{CH}_{2}\right), 28(\mathrm{CH}), 24\left(\mathrm{PhCH}_{3}\right), 19\left(\mathrm{CH}_{3}\right)_{2}$;

IR: $\left(\mathrm{cm}^{-1}\right)$ 3125-2856 (m, C-H), 2225 (m, CN), 1743 (s, C=O), 1634 (s, C=C), 1256 (s, CO-CH 3 ), 829, 756 (s, C-H out of plane). Anal. calcd. for $\mathrm{C}_{15} \mathrm{H}_{16} \mathrm{FNO}_{2}$ : C, 68.95; H, 6.17;

N, 5.36; Found: C, 64.82; H, 5.80; N, 4.97.

\subsubsection{Isobutyl 3-fluoro-4-methylphenylcyanoacrylate}

Yield $86 \%$; mp $114.3{ }^{\circ} \mathrm{C} ;{ }^{1} \mathrm{H}$ NMR $\delta 8.2(\mathrm{~s}, 1 \mathrm{H}, \mathrm{CH}=), 7.7,7.3(\mathrm{~m}, 3 \mathrm{H}, \mathrm{Ph}), 4.1(\mathrm{~d}, 2 \mathrm{H}$, $\left.\mathrm{CH}_{2}\right), 2.4\left(\mathrm{~s}, 3 \mathrm{H}, \mathrm{PhCH}_{3}\right), 2.0(\mathrm{~m}, 1 \mathrm{H}, \mathrm{CH}), 1.0\left(\mathrm{~d}, 6 \mathrm{H}, \mathrm{CH}_{3}\right) ;{ }^{13} \mathrm{C} \mathrm{NMR} \delta 162(\mathrm{C}=\mathrm{O})$, $159(\mathrm{HC}=), 154,134,131,121,114(\mathrm{Ph}), 114(\mathrm{CN}), 106(\mathrm{C}=), 73\left(\mathrm{CH}_{2}\right), 28(\mathrm{CH}), 21$ $\left(\mathrm{PhCH}_{3}\right), 19\left(\mathrm{CH}_{3}\right) ; \mathrm{IR}\left(\mathrm{cm}^{-1}\right): 2981(\mathrm{~m}, \mathrm{C}-\mathrm{H}), 2225(\mathrm{~m}, \mathrm{CN}), 1718(\mathrm{~s}, \mathrm{C}=\mathrm{O}), 1609$ (s, $\mathrm{C}=\mathrm{C}$ ), 1277 (s, C-O-CH ), 784, 752 (s, C-H out of plane). Anal. Calcd. for $\mathrm{C}_{15} \mathrm{H}_{16} \mathrm{FNO}_{2}$ : C, 68.95; H, 6.17; N, 5.36; Found: C, 67.09; H, 6.12; N, 5.13.

\subsubsection{Isobutyl 4-fluoro-2-methylphenylcyanoacrylate}

Yield 70.5\%; mp 75.2 ${ }^{\circ}$; ${ }^{1} \mathrm{H}$ NMR: $\delta 8.5(\mathrm{~s}, 1 \mathrm{H}, \mathrm{CH}=), 8.3-6.8(\mathrm{~m}, 3 \mathrm{H}, \mathrm{Ph}), 4.1(\mathrm{~d}, 2 \mathrm{H}$, $\left.\mathrm{CH}_{2}\right), 2.5\left(\mathrm{~s}, 3 \mathrm{H}, \mathrm{CH}_{3}\right), 2.1(\mathrm{~m}, 1 \mathrm{H}, \mathrm{CH}), 1.0\left(\mathrm{~d}, 6 \mathrm{H}, \mathrm{CH}_{3}\right) ;{ }^{13} \mathrm{C} \mathrm{NMR}: \delta 162(\mathrm{C}=\mathrm{O}), 152$ $(\mathrm{HC}=), 143,131,126,113(\mathrm{Ph}), 115(\mathrm{CN}), 104(\mathrm{C}=), 73\left(\mathrm{CH}_{2}\right), 28(\mathrm{CH}), 20\left(\mathrm{PhCH}_{3}\right), 19$ $\left(\mathrm{CH}_{3}\right)$; IR: (cm-1) 2987 (m, C-H), 2222 (m, CN), 1720 (s, C=O), 1597 (s, C=C), 1264 (s, C-O-CH ), 828, 752 (s, C-H out of plane). Anal. calcd. for $\mathrm{C}_{15} \mathrm{H}_{16} \mathrm{FNO}_{2}$ : C, 68.95; $\mathrm{H}$, 6.17; N, 5.36; Found: C, 68.17; H, 6.35; N, 5.53.

\subsubsection{Isobutyl 4-fluoro-3-methylphenylcyanoacrylate}


Yield 81.4\%; mp 73.5 ${ }^{\circ}$; ${ }^{1} \mathrm{H}$ NMR: $\delta 8.3$ (s, $\left.1 \mathrm{H}, \mathrm{CH}=\right), 7.9-6.7(\mathrm{~m}, 3 \mathrm{H}, \mathrm{Ph}), 4.2(\mathrm{~d}, 2 \mathrm{H}$,

$\left.\mathrm{CH}_{2}\right), 2.3\left(\mathrm{~s}, 3 \mathrm{H}, \mathrm{CH}_{3}\right), 2.1(\mathrm{~m}, 1 \mathrm{H}, \mathrm{CH}), 1.0\left(\mathrm{~d}, 6 \mathrm{H}, \mathrm{CH}_{3}\right) ;{ }^{13} \mathrm{C} \mathrm{NMR}: \delta 166(\mathrm{C}=\mathrm{O}), 154$ $(\mathrm{HC}=), 135,131,128,127(\mathrm{Ph}), 116(\mathrm{CN}), 102(\mathrm{C}=), 71\left(\mathrm{CH}_{2}\right), 26(\mathrm{CH}), 24\left(\mathrm{PhCH}_{3}\right), 18$ $\left(\mathrm{CH}_{3}\right)_{2}$; IR: $\left(\mathrm{cm}^{-1}\right)$ 3124-2883 (m, C-H), $2224(\mathrm{~m}, \mathrm{CN}), 1720$ (s, C=O), 1623 (s, C=C), 1246 (s, C-O-CH 3 ), 826 (s, C-H out of plane). Anal. calcd. for $\mathrm{C}_{15} \mathrm{H}_{16} \mathrm{FNO}_{2}$ : C, 68.95; H, 6.17; N, 5.36; Found: C, 64.82; H, 5.80; N, 4.97.

\subsubsection{Isobutyl 5-fluoro-2-methylphenylcyanoacrylate}

Yield 86\%; ${ }^{1} \mathrm{H}$ NMR $\delta 8.5(\mathrm{~s}, 1 \mathrm{H}, \mathrm{CH}=), 7.9-6.6(\mathrm{~m}, 3 \mathrm{H}, \mathrm{Ph}), 4.1\left(\mathrm{~d}, 2 \mathrm{H}, \mathrm{CH}_{2}\right), 2.3(\mathrm{~s}$, $\left.3 \mathrm{H}, \mathrm{PhCH}_{3}\right), 2.1(\mathrm{~m}, 1 \mathrm{H}, \mathrm{CH}), 1.0\left(\mathrm{~d}, 6 \mathrm{H}, \mathrm{CH}_{3}\right) ;{ }^{13} \mathrm{C} \mathrm{NMR} \delta 167(\mathrm{C}=\mathrm{O}), 152(\mathrm{HC}=)$, 148, 135, 133, 131, 119, $113(\mathrm{Ph}), 115(\mathrm{CN}), 103(\mathrm{C}=), 73\left(\mathrm{CH}_{2}\right), 28(\mathrm{CH}), 20\left(\mathrm{CH}_{3}\right), 19$ $\left(\mathrm{CH}_{3}\right)_{2}$; IR $\left(\mathrm{cm}^{-1}\right): 3557-2884(\mathrm{~m}, \mathrm{C}-\mathrm{H}), 2242(\mathrm{~m}, \mathrm{CN}), 1742(\mathrm{~s}, \mathrm{C}=\mathrm{O}), 1672(\mathrm{~s}, \mathrm{C}=\mathrm{C})$, 1227 (s, C-O-CH3), 822, 743 (s, C-H out of plane). Anal. Calcd. for $\mathrm{C}_{15} \mathrm{H}_{16} \mathrm{FNO}_{2}$ : C, 68.95; H, 6.17; N, 5.36; Found: C, 66.99; H, 6.18; N, 5.93.

\subsubsection{Isobutyl 4-fluoro-3-phenoxyphenylcyanoacrylate}

Yield $87 \%$; mp $128.1{ }^{\circ} \mathrm{C} ;{ }^{1} \mathrm{H}$ NMR $\delta 8.5(\mathrm{~s}, 1 \mathrm{H}, \mathrm{CH}=), 7.9-6.5(\mathrm{~s}, 8 \mathrm{H}, \mathrm{Ph}), 4.1(\mathrm{~s}, 2 \mathrm{H}$, $\left.\mathrm{CH}_{2}\right), 2.0(\mathrm{~m}, 1 \mathrm{H}, \mathrm{CH}), 1.0\left(\mathrm{~d}, 6 \mathrm{H},\left(\mathrm{CH}_{3}\right)_{2} ;{ }^{13} \mathrm{C} \mathrm{NMR} \delta 162(\mathrm{C}=\mathrm{O}), 153(\mathrm{HC}=), 145,130\right.$, 129, 127, 124, 123, $118(\mathrm{Ph}), 115(\mathrm{CN}), 98(\mathrm{C}=), 73\left(\mathrm{CH}_{2}\right), 28(\mathrm{CH}), 19\left(\mathrm{CH}_{3}\right)_{2}$; IR $\left(\mathrm{cm}^{-}\right.$ 1): 2966 (m, C-H), 2222 (m, CN), 1725 (s, C=O), 1588 (s, C=C), 1274 (s, C-O-CH3), 846, 751 (s, C-H out of plane). Anal. Calcd. for $\mathrm{C}_{20} \mathrm{H}_{18} \mathrm{FNO}_{3}: \mathrm{C}, 70.78 ; \mathrm{H}, 5.35 ; \mathrm{N}, 4.13$;

Found: C, 70.40; H, 5.40; N, 4.11. 


\subsection{Homopolymerization}

An attempted homopolymerization of the IPCA compounds in the presence of ABCN did not produce any polymer as indicated by the lack of a precipitate in methanol. The inability of the monomers to polymerize is associated with steric difficulties encountered in homopolymerization of 1,1- and 1,2-disubstituted ethylenes [14]. Homopolymerization of ST under conditions identical to those in copolymerization experiments yielded $18.3 \%$ of polystyrene, when polymerized for $30 \mathrm{~min}$.

\subsection{Synthesis and characterization of styrene - IPCA copolymers}

Copolymers of the styrene (ST) and the IPCA compounds, P(ST-co-IPCA) were prepared in 25-mL glass screw cap vials at ST/IPCA $=3(\mathrm{~mol})$ the monomer feed using $0.12 \mathrm{~mol} / \mathrm{L}$ of $\mathrm{ABCN}$ at an overall monomer concentration $2.44 \mathrm{~mol} / \mathrm{L}$ in $10 \mathrm{~mL}$ of toluene. The copolymerization was conducted at $70^{\circ} \mathrm{C}$. After a predetermined time, the mixture was cooled to room temperature, and precipitated dropwise in methanol. The composition of the copolymers was determined based on the nitrogen content. The novel synthesized IPCA compounds copolymerized readily with ST under free-radical conditions (Scheme 2) forming white flaky precipitates when their solutions were poured into methanol. The conversion of the copolymers was kept between 10 and $20 \%$ to minimize compositional drift (Table 1). 
Table 1. Copolymerization of isobutyl phenylcyanoacrylates with styrene.

\begin{tabular}{|l|c|c|c|c|}
\hline \multicolumn{1}{|c|}{$\mathrm{R}$} & $\begin{array}{c}\text { Yield }^{\mathrm{a}} \\
(\mathrm{wt} \%)\end{array}$ & $\begin{array}{c}\mathrm{N} \\
(\mathrm{wt} \%)\end{array}$ & $\begin{array}{c}\mathrm{m}_{1} \text { in } \\
\text { copol. } \\
(\mathrm{mol} \%)\end{array}$ & $\begin{array}{c}\mathrm{m}_{2} \text { in } \\
\text { copol. } \\
(\mathrm{mol})\end{array}$ \\
\hline $2-\mathrm{F}-5-\mathrm{CH}_{3}$ & 11.4 & 1.72 & 84.2 & 15.8 \\
\hline $2-\mathrm{F}-6-\mathrm{CH}_{3}$ & 11.3 & 1.90 & 82.1 & 17.9 \\
\hline $3-\mathrm{F}-2-\mathrm{CH}_{3}$ & 12.7 & 1.98 & 81.1 & 18.9 \\
\hline $3-\mathrm{F}-4-\mathrm{CH}_{3}$ & 13.5 & 2.66 & 71.8 & 28.2 \\
\hline $4-\mathrm{F}-2-\mathrm{CH}_{3}$ & 12.3 & 2.46 & 74.8 & 25.2 \\
\hline $4-\mathrm{F}-3-\mathrm{CH}_{3}$ & 14.1 & 1.91 & 81.9 & 18.1 \\
\hline $5-\mathrm{F}-2-\mathrm{CH}_{3}$ & 13.7 & 9.52 & 72.6 & 27.4 \\
\hline $4-\mathrm{F}-3-\mathrm{C}_{6} \mathrm{H}_{5} \mathrm{O}$ & 12.7 & 2.52 & 67.6 & 32.4 \\
\hline
\end{tabular}

Nitrogen elemental analysis showed that between 22.4 and $41.1 \mathrm{~mol} \%$ of IPCA is present in the copolymers, which is indicative of relatively high reactivity of the IPCA monomers towards ST radical which is typical of oxy ring-substituted different esters PCA [16-20]. Since IPCA monomers do not homopolymerize, the most likely structure of the copolymers would be isolated IPCA monomer $(\mathrm{y}=1)$ units alternating with short ST sequences $(\mathrm{x}>1)($ Scheme 2$)$.

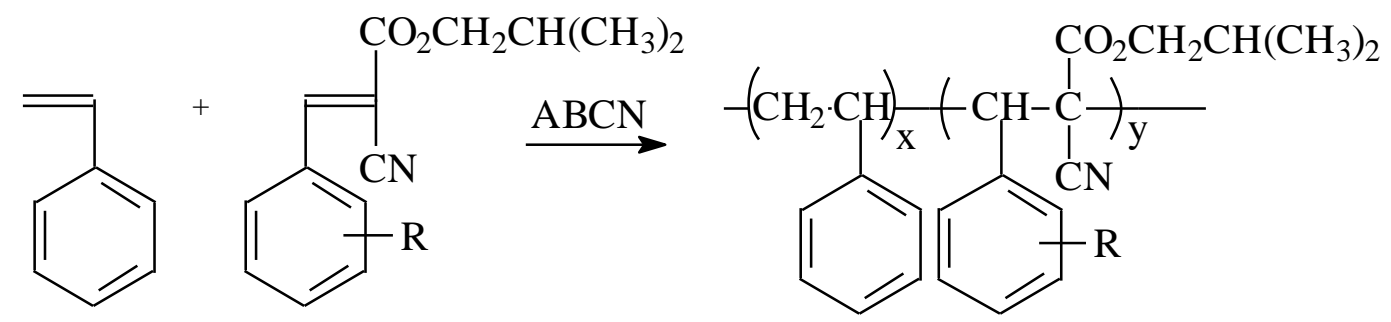

Scheme 2. Copolymerization of ST and the ring-substituted isobutyl

phenylcyanoacrylates, $\mathrm{RPhCH}=\mathrm{C}(\mathrm{CN}) \mathrm{CO}_{2} \mathrm{CH}_{2} \mathrm{CH}\left(\mathrm{CH}_{3}\right)_{2}, \mathrm{R}=$ 2-fluoro-5-methyl, 2-

fluoro-6-methyl, 3-fluoro-2-methyl, 3-fluoro-4-methyl, 4-fluoro-2-methyl, 4-fluoro-3methyl, 5-fluoro-2-methyl, 4-fluoro-3-phenoxy. 
The copolymers prepared in the present work are all soluble in ethyl acetate, THF, DMF and $\mathrm{CHCl}_{3}$ and insoluble in methanol, ethyl ether, and petroleum ether.

\section{Conclusions}

Novel fluoro, methyl, and phenoxy ring-disubstituted isobutyl phenylcyanoacrylates were prepared and copolymerized with styrene. The compositions of the copolymers were calculated from nitrogen analysis.

\section{Acknowledgments}

The authors are grateful to acknowledge that the project was partly supported by Chicago Society of Coating Technology (CSCT).

\section{References}

[1] Klemarczyk, P.; Guthrie, J. Advances in anaerobic and cyanoacrylate adhesives. In Advances in Structural Adhesive Bonding, 1st ed.; Dillard, D., Ed.; Woodhead Publishing Limited: Cambridge, UK, 2010; pp. 96-131, ISBN 978-1-84569-435-7.

[2] Shantha, K.L.; Thennarasu, S.; Krishnamurti, N. Developments and applications of cyanoacrylate adhesives. J. Adhes. Sci. Technol. 1989, 3, 237-260. 
[3] J.M. Korde and B. Kandasubramanian, Biocompatible alkyl cyanoacrylates and their derivatives as bio-adhesives, Biomaterials Science, 10.1039/C8BM00312B, 6, 7, (1691-1711), (2018).

[4] Zaki MEA, Fathalla OA, Swelam SA, Aly HF (2004) Synthesis of pyrido[2,1c][1,2,4]triazine, 1,2,4-triazolo[4,3-a]pyridine and 2-(pyrazolyl)nicotinonitrile and their effect on Biomphalaria alexandrina snail enzymes. Acta Poloniae Pharmaceutica 61: $55-64$.

[5] Matsuoka M, Takao M, Kitao T, Fujiwara T, Nakatsu K (1990) Cyanovinylheteroaromatics for Organic Nonlinear Optics. Molecular Crystals and Liquid Crystals, 182A: 71-79.

[6] Burate PA, Javle BR, Desale PH, Kinage AK (2019) Amino acid amide based ionic liquid as an efficient organo-catalyst for solvent-free Knoevenagel condensation at room temperature. Catalysis Letters 149(9): 2368-2375.

[7] Medyouni R, Hamdi N, Ben Said R, Al-Ayed AS, Zagrouba F (2013) Clean procedure and DFT study for the synthesis of 2-amino-3-ethoxycarbonyl-4-(aryl)-4Hpyrano-[3,2-c]-chromene-5-ones derivatives: A novel class of potential antimicrobial and antioxidant agents. Journal of Chemistry 2013: 1-9.

[8] Hassan AY, Sarg MT, Hussein EM (2019) Design, synthesis and anticancer activity of novel benzothiazole analogues. Journal of Heterocyclic Chemistry 56(4): 14371457. 
[9] Moacyr JB, De Melo R, Wanessa LB, De Sena, RO, De Moura, Iris TT et al. (2017)

Synthesis and anticancer evaluation of thiazacridine derivatives reveals new selective molecules to hematopoietic neoplastic cells. Combinatorial Chemistry \& High Throughput Screening 20(8): 713.

[10] Meng D, Qiao Y, Wang X, Wen W, Zhao S (2018) DABCO-catalyzed

Knoevenagel condensation of aldehydes with ethyl cyanoacetate using hydroxy ionic liquid as a promoter. RSC Advances 8(53): 30180-30185.

[11] Brica S, Freimane L, Kulikovska L, Zicmanis A (2017) N,N'-dialkylimidazolium dimethyl phosphates - promising media and catalysts at the same time for condensation reactions. Chemical Science International Journal 19(4): 1-9.

[12] Bhuiyan M, Mosharef H, Rahman KM, Alam MA, Mahmud M (2013) Microwave assisted Knoevenagel condensation: synthesis and antimicrobial activities of some $\alpha$-cyanoacrylates. Pakistan Journal of Scientific and Industrial Research, Series A: Physical Sciences 56(3): 131-137.

[13] Nowicka A, Liszkiewicz H, Nawrocka WP, Wietrzyk J, Kempinska K, Drys A (2014) Synthesis and antiproliferative activity in vitro of new 2-aminobenzimidazole derivatives. Reaction of 2-arylideneaminobenzimidazole with selected nitriles containing active methylene group. Central European Journal of Chemistry 12(10): 1047-1055.

[14] Odian, G. Principles of Polymerization, 4th Ed., Wiley-Interscience: New York, 2004. 
[15] Hall, H. K., Jr.; Padias, A. B. J. Polym. Sci.Part A: Polym. Chem. 2004, 42, 2845-2858.

[16] Hall, H. K. Jr.; Ykman, P. Macromolecules. 1977, 10, 464.

[17] Kharas, G. B. Trisubstituted Ethylene Copolymers. In Polymeric Materials Encyclopedia, Salamone, J.C., (Ed.) CRC Press: Boca Raton, FL, 11, 8405, 1996.

[18] Novel Copolymers of Styrene. 14. Some Ring-Disubstituted Methyl 2-Cyano-3Phenyl-2-Propenoates. G.B. Kharas, A. Shahbein, J.L. Fabbri, C.E. Fanter, D.R. Garcia, M.G. Ibarra, D. Lane, E. Marshall, E.E. Pierce, I.F. Sanchez, B.C. Sandoval, and A.D. Santi. J. Macromol. Sci. A51(5) 394-398 (2014).

[19] Novel Copolymers of Styrene and Dialkoxy Ring-substituted Methyl 2-cyano-3phenyl-2-propenoates. G.B. Kharas, J.L. Christensen, D.J. Cichanski, K.E. Goldman, C.L. Gordon, L.M. Knowles, C.N. Krefft, M. Matouk, and K. Watson. J. Macromol. Sci., 43 (7) 989-994 (2006).

[20] Novel Copolymers of Styrene. 4. Alkyl Ring-substituted Ethyl 2-Cyano-3-phenyl-2propenoates. G.B. Kharas, E.S. Molina, E.E. Pierce, S.A.B. Cocjin, C. Cruz, K.M. Fair, S.S. Flaksman, M.J. Liggins, A.D. Meglei, M.E. Pantos, and G.C. Pisano. J.Macromol. Sci. A50 (2) 144-148 (2013).

[21] Novel Copolymers of Styrene. 6. Alkoxy Ring-substituted Ethyl 2-Cyano-3-phenyl2-propenoates.G.B. Kharas, A.A. Delgado, N.E. Anderson, A. Bajor, A.C. Colbert, A. Coleman, C.E. Gregory, J. Hayes, J. Lantin, J.M. Malecki, T.C. Murphy, A. 
Oprescu, B.F. Rydzon, and I. Timoshevskaya. J.Macromol. Sci. A50 (3) 276-280 (2013).

[22] Novel Copolymers of Styrene. 2. Some Ring-substituted Ethyl 2-Cyano-3-phenyl-2propenoates. G. B. Kharas, E.S. Molina, B. Bobot, J. Bueno, J. Carney, J.Y.C. Chung, K.N. Krerowicz, T. Miller, S.L.I. Mills, M.A. Stankovich, I. Syed, C.S. Vaidya, and P. Wesolowski. J. Macromol. Sci. A50 (1) 1-5 (2013).

[23] Novel Copolymers of Styrene. 5. Alkyl and Alkoxy Ring-Disubstituted Propyl 2Cyano-3-Phenyl-2-Propenoates. G.B. Kharas, W.M.B. Barros, A. Affaneh, L.A. Alyahya, A.S. Ansari, M.J. Asztalos, P.T. Burns, Y. Cardoza, K.J.O. Galvan, K.J. Hall, S.M. Short, and M.A. Sovereign. J. Macromol. Sci. A53(11) 664-668 (2016).

[24] Novel Copolymers of Styrene. 6. Some Ring-Disubstituted Propyl 2-Cyano-3Phenyl-2-Propenoates. G.B. Kharas, L.A. Alyahya, S. M. Rocus, A. Ismail, R. Juarez, A. Kavaliauskaite, K.Y. Lechuga, A.C. Leeper, and G.C. Lenti. J. Macromol. Sci. A53(12) 725-728 (2016).

[25] Synthesis and styrene copolymerization of dimethyl and dimethoxy ring-substituted isopropyl cyanophenyl propenoates. R.L. Pride, A. MacDonald, E.E. McCarthy, A.N. Modi, E. Mucciolo, A.L. Schinderle, S. Trewartha, J.D. Tylka, M. Wade, J. Wat, W.S. Schjerven and G.B. Kharas. Academic J Polym Sci. 3(1): 555602 (2019). DOI:10.19080/AJOP.2019.02.555602

[26] Synthesis and styrene copolymerization of novel oxy ring-disubstituted isopropyl phenylcyanoacrylates. J.L. Zepeda, M.M. Betts, S. Dalloul, A.G. Gonzalez, J.K. 
Henning, R.X. Liu, J.J. Ludtke, J.R. Mihai, S.M. Rocus, W.S. Schjerven, and G.B. Kharas. J. Phys. Chem. Res. 1(2) 1-5 (2019).

[27] Synthesis and styrene copolymerization of novel ring-disubstituted isopropyl cyanoacrylates. P.M. Whelpley, S. Bajramovic, D.M. Bracamontes, G.A. Buechner, A.D. Eremin, E.J. Kowalczyk, D.D. Lender, R. McCann, W.S. Schjerven, G.B. Kharas. ChemRxiv (2019), 1-15. https://doi.org/10.26434/chemrxiv.9891161.v1.

[28] Novel Copolymers of Styrene. 6. Some Ring-Disubstituted Butyl 2-Cyano-3-Phenyl2-Propenoates. G.B. Kharas, S.M. Rocus, M. Zuziak, V. Elangovan, A.N. Kovaleva, S. Malik, O. Nwosu, L.A. Patel, A. Piche, and S.J. Rosengarden. J. Macromol. Sci. A52 (12) 971-975 (2015).

[29] Novel Copolymers of Styrene. 5. Methyl and Methoxy Ring-Disubstituted Butyl 2Cyano-3-Phenyl-2-Propenoates. G.B. Kharas, H. Feng, L.A. Arendt, S.E. Belton, M.Q. Edwards, E.K. Franz, A. Grin, C.M. Hale, I.C. Oyeyipo, A.L. Wolske, P.L. Zavala, and A. Zenunovic. J. Macromol. Sci. A52 (12) 976-981 (2015).

[30] One-pot esterification and amide formation via acid-catalyzed dehydration and Ritter reactions. Dawar, Pankaj; Raju, M. Bagavan; Ramakrishna, Ramesha Andagar. Synthetic Communications (2014), 44(6), 836-846.

[31] Smith, M. B.; March, J. Addition to Carbon-Hetero Multiple Bonds, In March's Advanced Organic Chemistry, J. Wiley \& Sons: New York, Ch.16, 1225 (2001). 